\title{
Subestimación del peso corporal en escolares mexicanos
}

\section{Underestimation of body weight in Mexican school children}

\author{
Romana Silvia Platas Acevedo ${ }^{1}$, Gilda Gómez-Peresmitrél \\ y Gisela Pineda García ${ }^{2}$
}

Citación: Platas A., R.S., Gómez-Peresmitré, G. y Pineda G., G. (2019). Subestimación del peso

corporal en escolares mexicanos. Psicología y Salud, 29(2), 269-277.

\section{RESUMEN}

El objetivo principal fue detectar el grado de discordancia o concordancia entre el índice de masa corporal y la propia percepción del peso en una muestra de niños con sobrepeso y obesidad. Se trabajó con una muestra no probabilística de 549 escolares de primaria de la Ciudad de México con peso corporal elevado: 284 varones y 265 mujeres, cuyo rango de edad fue de 9 a 12 años, a quienes, con el consentimiento de sus padres, se aplicó una batería de pruebas con propiedades psicométricas aceptables. Las relaciones más importantes en el modelo de factores de riesgo para las niñas fueron insatisfacción e índice de masa corporal, insatisfacción y subestimación, y subestimación e índice de masa corporal, mientras que para los niños fueron subestimación e índice de masa corporal, percepción de amigos e índice de masa corporal, y percepción de amigos e insatisfacción. Se concluye que valores extremos del índice de masa corporal se relacionan con insatisfacción corporal, esto es, el deseo de ser más delgado, lo que conduce a la subestimación del peso. Se señala que tal subestimación contribuye a que se mantenga ese peso, lo que la vuelve un factor de riesgo que debe tomarse en cuenta en los programas de prevención que se desarrollan en el país.

Palabras clave: Sobrepeso y obesidad; Escolares mexicanos; Índice de masa corporal; Subestimación corporal; Modelos estructurales.

\begin{abstract}
The main objective of this study was to examine the association between body mass index and self-perceived body weight in a sample of overweight and obese children. A non-probabilistic sample of 549 overweight elementary school children from Mexico City: 284 boys and 265 girls, aged 9 to 12, responded to a battery of tests with acceptable psychometric properties, after informed consent by their parents. The most important risk factor associations for girls were body dissatisfaction with body mass index, dissatisfaction with underestimation, and underestimation with body mass index. For boys these included underestimation with body mass index, perception of friends with body mass index, and perception of friends with dissatisfaction. It is concluded that extreme values of body mass index are related to body dissatisfaction (desire to be thinner), which leads to underestimation of body weight. Underestimation of the body weight contributes to maintaining excessive weight, thus becoming a risk factor to be taken into account while developing prevention programs.
\end{abstract}

Key words: Overweight; Obesity; Mexican scholars; Body mass index; Body weight underestimation; Structural models.

\footnotetext{
${ }^{1}$ División de Investigación y Estudios de Postgrado, Facultad de Psicología, Universidad Nacional Autónoma de México, Edificio D, Cubículo 11, Mezzanine. Av. Universidad 3004, Col. Copilco-Universidad, Del. Coyoacán, 04510 México, D.F., tel. (55)56-22-22-52, correos electrónicos: romsip@unam.mx y gildag@unam.mx. Artículo recibido el 23 de enero y aceptado el 8 de marzo de 2018.

${ }^{2}$ Facultad de Medicina y Psicología, Universidad Autónoma de Baja California, Calzada Universidad 14418, Parque Internacional Industrial Tijuana, 22390 Tijuana, B.C., México, tel. (664)682-12-33, correo electrónico:gispineda@uabc.edu.mx.
} 


\section{INTRODUCCIÓN}

$\mathrm{L}$ os altos costos económicos y sociales que se derivan de las comorbilidades que trae consigo la obesidad hacen de la misma la pandemia más grave de esta época y el problema más difícil de abordar por la diversidad de factores que intervienen en su génesis y su carácter crónico y recidivante (Barquera et al., 2010; Zamudio y Muñoz, 2016). La obesidad se define como la acumulación anormal o excesiva de grasa con relación a la edad y sexo, resultado del desequilibrio entre la ingestión y el gasto de energía (Organización Mundial de la Salud, 2016). En México, el rápido aumento de los porcentajes de personas obesas (adultos y niños) es alarmante, ya que en menos de medio siglo se ha convertido en un grave problema de salud pública, conjuntamente con las enfermedades crónicas no transmisibles (Córdoba, 2016).

En la búsqueda de los factores que intervienen en el desarrollo de la patología pandémica de la obesidad, Madrigal et al. (1999), Madrigal et al. (2000), Atalah, Urteaga y Rebolledo (2004) y Mújica et al. (2009) señalan que la percepción errónea que el individuo tiene de su imagen o tamaño corporal influye o se relaciona directamente con su estado nutricional. Gómez-Peresmitré, Jaëger, Pineda y Platas, (2012) y Gómez-Peresmitré, Platas y Pineda (2015) se han enfocado en la hipótesis que propone que el índice de masa corporal (IMC en lo sucesivo), en sus valores extremos (emaciación y obesidad) y asociado con la insatisfacción corporal, conducirá a la alteración del peso corporal. Al respecto, se ha encontrado que en el caso de la emaciación (IMC < 16) y la insatisfacción positiva (deseo de estar más delgado), se produce sobreestimación. Las mujeres con bajo peso se perciben a sí mismas con más peso del que realmente tienen (Gómez-Peresmitré et al., 2012; Gómez-Peresmitré et al., 2015; Míguez, De la Montaña, González y González, 2011), lo que es muy evidente en una condición patológica como la anorexia nerviosa, en la que la mujer emaciada se percibe con sobrepeso. En el caso de la obesidad (IMC $\geq 25$ ), tal asociación conduce a una subestimación (Gómez-Peresmitré et al., 2012; Matus, Álvarez, Nassar y Mondragón, 2016). Es- tos patrones de relaciones tríádicas IMC-insatisfacción-alteración corporal deben atenderse prioriariamente durante las intervenciones preventivas de la obesidad (Gómez-Peresmitré, Platas, Pineda y Guzmán, en revisión).

Debe también analizarse la percepción de los padres. Revisiones previas han mostrado que más de la mitad de los padres no perciben correctamente el peso corporal de sus hijos (Chen, Binns, Zhao y Liu, 2014; Lundahl, Kidwell y Nelson, 2014; Park, 2014; Pasch et al., 2016; West et al., 2008; Zonana y Conde, 2010), explicándolo en parte el hecho de que lo ignoran, así como su desconocimiento objetivo de qué es la obesidad (cuando se trata del peso normal, en qué punto de corte se puede hablar ya de sobrepeso, etc.). Vuorela, Saha y Salo (2010) y Park (2014) han demostrado que hay una mayor prevalencia de la subestimación del peso corporal de los hijos cuando uno de los padres o ambos son obesos. La mayoría de las madres que consideran que sus hijos tienen un peso normal y que son saludables no obstante su indudable obesidad, evitan reconocer que tienen un problema de salud, pues un hijo sano indica que son buenas madres (Vuorela et al., 2010). En este mismo sentido, la extendida creencia de que un niño "gordito" es un modelo de buena salud representa para los progenitores la confirmación de que han desempeñado de un modo excelente su labor de padres (Angarita, Martínez, Rangel, Betancourt y Prada, 2012; Chávez et al., 2016), evadiendo así su falta de responsabilidad y el descontrol que tienen sobre el comportamiento alimentario del niño (Allen y Prkachin, 2013; Guzmán, 2012).

En el caso de la subestimación del peso corporal, se deben tomar en cuenta los antecedentes culturales, las tradiciones, el país de origen y la educación. Un ejemplo de ello es el significado y el papel que desempeña la comida entre las madres mexicanas, quienes la equiparan con el amor debido a que en su mayoría la utilizan como un premio o como un castigo según la ocasión, dándole así un significado connotativo diferente e incluso opuesto (Guzmán, 2012; Platas 2014; Zonana y Conde, 2010). Otra posible explicación de la subestimación del peso corporal entre las personas obesas es que se trata de un fenómeno protector 
ante una sociedad "obesofóbica" que estigmatiza la corpulencia y hace a las personas que la padecen el blanco de sus críticas; por ello, las personas obesas normalizan su exceso de peso corporal y su mal comportamiento alimentario, lo que limita la eficacia de los esfuerzos para prevenir dicha condición (Gómez-Peresmitré, 1997; Platas, 2014).

Britek-Matera (2011) y Míguez et al. (2011) señalan que entre los varones adolescentes se ha encontrado una mayor frecuencia de subestimación del peso corporal que entre las adolescentes mujeres, aunque éstas tienden más a subestimarlo o a sobreestimarlo. Asimismo, se ha visto que las mujeres más insatisfechas con su imagen corporal son aquellas que padecen estados nutricionales carenciales -como desnutrición- o sobrepeso y obesidad (Jáuregui, Bolaños, Santiago y Garrido, 2011); en el caso de los hombres, los que se encuentran más insatisfechos son aquellos con exceso de peso.

La complejidad del tratamiento de la obesidad requiere un abordaje multidisciplinario que implica un cambio en el estilo de vida, lo que es difícilmente alcanzable si no hay conciencia de la enfermedad. Se ha afirmado que conocer cómo se perciben las personas con sobrepeso y obesidad ayudará a definir nuevas estrategias que fortalezcan la necesidad de tener un peso saludable (Atalah et al., 2004).

Siguiendo esa perspectiva, se planteó como el propósito de la presente investigación detectar en una muestra de niños con sobrepeso y obesidad el grado de discordancia o concordancia (alteración del peso corporal) entre el IMC y la propia percepción del peso, evaluando de esta manera dicha percepción subjetiva (subestimación) y buscando con ello contribuir al desarrollo de nuevas estrategias para prevenir la obesidad.

\section{MÉTODO}

\section{Participantes}

La muestra total no probabilística e intencional quedó formada por 1,381 escolares de quinto y sexto grados de primaria, provenientes de siete escuelas públicas y privadas de la Ciudad de México y su zona conurbada, quienes decidieron par- ticipar en la investigación. De ellos, se seleccionó a 549 escolares con IMC elevado de los citados grados, los cuales se subdividieron del siguiente modo: $n 1$ : 284 con peso corporal elevado (129 con sobrepeso y 155 con obesidad), y $n 2: 265$ niñas con peso corporal elevado (162 con sobrepeso y 103 con obesidad). Los participantes se distribuyeron en un rango de edad de 9 a 12 años $(\mathrm{M}=11.2$ años y D.E. $=.72)$. Se clasificó como obesos a los participantes con un IMC-P (centil = 95), y con sobrepeso a aquellos con IMC-P $($ centil $=85<95)$.

\section{Variables e instrumentos}

Cuestionario de Alimentación y Salud para Preadolescentes (Gómez-Peresmitré, 2001). Se aplicó este instrumento, cuyo coeficiente alfa de Cronbach es de .80, para explorar las siguientes variables: percepción de los padres, percepción de los amigos y percepción de la ingesta alimentaria.

Escala de Factores de Riesgo Asociados con Trastornos de la Alimentación (EFRATA-II) (Platas y Gómez-Peresmitré, 2013). La dieta crónica y restringida se evaluó mediante esta escala, cuyo promedio se obtiene con los puntajes de los cinco ítems que conforman el factor 2. Cuenta con un coeficiente alfa de Cronbach $(\alpha)$ de .83 .

Índice de masa corporal (IMC). Se obtiene dividiendo el peso sobre la talla, elevada ésta al cuadrado (peso/talla ${ }^{2}$ ). Para la obtención del peso, se utilizó una báscula profesional de precisión marca Tanita, Modelo 553, y para la talla un estadiómetro portátil marca Seca, Modelo 214, con un rango de medición de 2-207 cm y divisiones de $1 \mathrm{~mm}$. Debido a la edad de los participantes, se consideraron los puntos de corte percentilar establecidos por el National Center for Health Statistics (2003).

Satisfacción o Insatisfacción con la Imagen Corporal ( $\alpha$ de .80) (Gómez-Peresmitré, 2001). La satisfacción o insatisfacción con la imagen corporal se mide mediante una escala visual de seis siluetas que cubren un continuo de peso corporal que va de una muy delgada (puntuación $=1$ ) a una muy gruesa u obesa (puntuación $=6$ ), pasando por una de peso normal (puntuación $=3$ ). Para evaluar la imagen corporal se presenta la escala en dos oca- 
siones diferentes y con las siluetas ordenadas de manera aleatoria en cada ocasión, buscando así la figura real ("Mi cuerpo se parece más a...") e ideal ("Me gustaría que mi cuerpo fuera cómo...") del participante.

Alteración de la Imagen Corporal ( $\alpha$ de .80) (Gómez-Peresmitré, 2001). Mediante esta escala se estiman dos variables: autopercepción del peso corporal (variable subjetiva) y peso real IMC (variable objetiva). Se establece a partir de la respuesta a dos ítems: "De acuerdo a la siguiente escala, tú te consideras..." e "Índice de masa corporal". Dicha escala está compuesta de cinco opciones de respuesta, que van de muy delgada(o) (1) a obesa(o) o muy gorda(o) (5). La diferencia positiva o negativa entre las puntuaciones de estos ítems (autopercepción del peso corporal-IMC) proporciona el puntaje de sobreestimación o subestimación, respectivamente; una diferencia igual a 0 se interpreta como no alteración del peso corporal.

Actividad e inactividad fisica. Esta variable se define como el promedio de los puntajes totales dados a los ítems del Cuestionario de Actividad e Inactividad Física de los Estudiantes Mexicanos (CAINM) (Hernández et al., 2000) ( $\alpha$ de .80), que incluye la evaluación de la actividad física moderada o vigorosa y la inactividad física.

\section{Procedimiento}

Se capacitó a estudiantes de Psicología en la aplicación del instrumento, así como de Nutrición para la toma del peso y la talla. Tras acordar la aplicación con los directivos y profesores de las escuelas, y una vez obtenido previamente el consentimiento informado de los participantes y de sus padres, ésta se realizó grupalmente en una sola sesión de 60 minutos. Se siguieron las recomendaciones éticas de la Sociedad Mexicana de Psicología (2010) para procedimientos no intrusivos en la investigación.

\section{Análisis estadísticos}

Los datos (distribuciones de las variables) se analizaron mediante el uso del paquete estadístico
SPSS, versión 20. Para el modelamiento de ecuaciones estructurales se utilizó el programa informático Amos, versión 6, de Windows XP; la evaluación de los modelos se efectuó con el método de máxima verosimilitud (Arbuckle y Wothke, 1999), y para el contraste de los modelos estructurales se emplearon cuatro índices de ajuste $\chi^{2}$ no significativo, con valores AGFI y NFI de $.95 \mathrm{y}$ RMSEA $<.05$.

\section{RESULTADOS}

\section{Satisfacción e insatisfacción con la imagen corporal}

Para obtener la satisfacción o insatisfacción con la imagen corporal, se obtuvo la diferencia entre las puntuaciones de la figura actual menos las de la figura ideal. La Figura 1 muestra la distribución porcentual de esta variable según la correspondiente categoría de IMC-P.

Figura 1. Distribución porcentual de la variable satisfacción/insatisfacción de la imagen corporal por sexo y categoría de IMC-P.

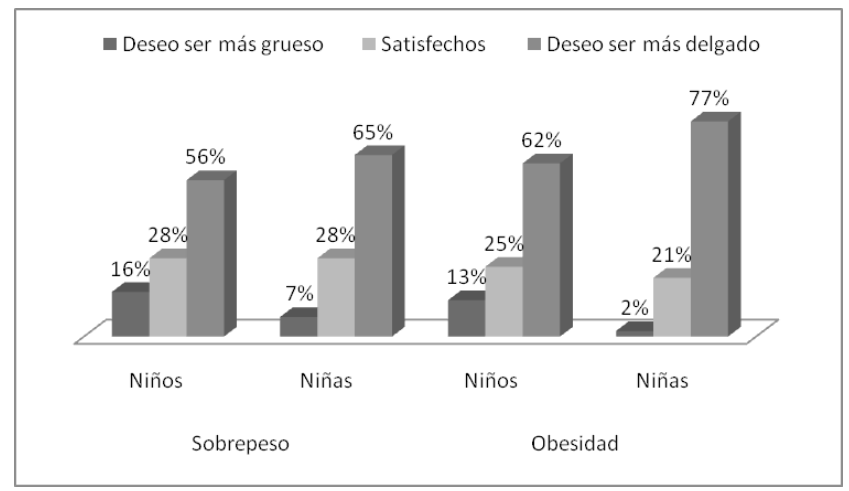

Debe señalarse que las niñas con sobrepeso y obesidad fueron las más insatisfechas con su peso, queriendo ser más delgadas (65 y 77\%, respectivamente), en comparación con 56 y $62 \%$ de los niños; $16 \%$ de los niños con sobrepeso y $13 \%$ con obesidad deseaban ser más gruesos, a diferencia de las niñas, que mostraron porcentajes menores (7 y $2 \%$, respectivamente). En porcentajes similares de 20 a $28 \%$ los participantes mostraron satisfacción, independientemente de su sexo y del 
peso, en tanto que las niñas obesas mostraron el porcentaje más alto en su deseo de querer ser más delgadas $(77 \%)$.

\section{Alteración de la imagen corporal}

Respecto a la alteración de la imagen corporal, puede observarse en la Figura 2 que los niños del grupo con obesidad, comparados con el de sobrepeso, obtuvieron mayores porcentajes de subestimación (96 vs. 71\%), al igual que las niñas con sobrepeso y obesidad (87 vs. $77 \%$, respectivamente).

Figura 2. Distribución porcentual de la variable alteración.

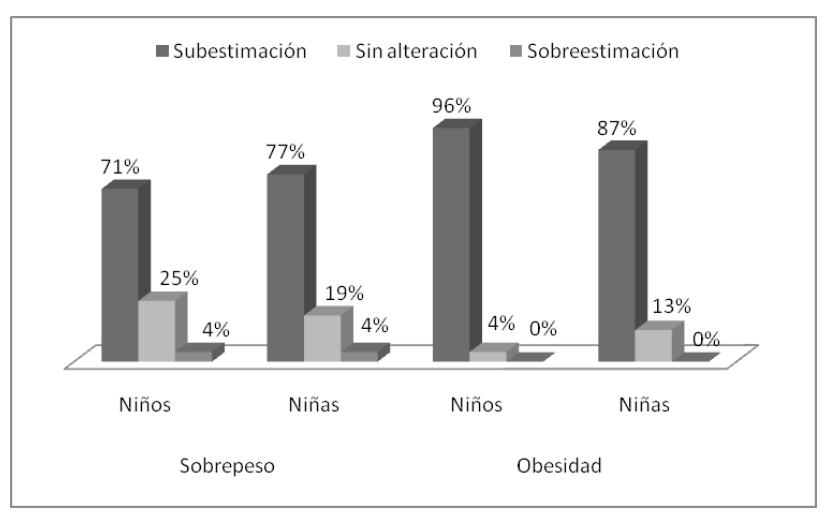

\section{Modelos estructurales}

Con el propósito central de entender y explicar el papel que desempeña el IMC (variable dependiente) en relación con los factores de riesgo para la génesis y mantenimiento de la obesidad, se probaron diversos modelos con las variables de carácter psicológico y sociocultural de acuerdo con su pertinencia teórica y empírica, para lo cual se integró la información recabada a través de tres elementos básicos: literatura especializada, valores de correlaciones entre las variables y modelos generados a partir de regresiones lineales. Como ya se indica líneas atrás, para el contraste de los modelos estructurales se utilizaron cuatro índices de ajuste: RMSEA, AGFI, NFI y $\chi^{2}$.

El modelo estructural predictivo para las niñas con IMC elevado (P85-P95 y $p=95)$, esto es, niñas con sobrepeso y obesidad, resultó con niveles adecuados de bondad de ajuste: $\chi^{2}$ no significativa (no obstante el tamaño grande de la muestra) y valores AGFI y NFI $=.95$ y RMSEA $<.05$. En la Figura 3 pueden observarse correlaciones bajas (ligas con doble flecha), prácticamente inexistentes, hallándose la más alta entre "percepción de amigos" y "percepción de los padres" $(r=.49)$.

Figura 3. Modelo de factores de riesgo en niñas con sobrepeso y obesidad.

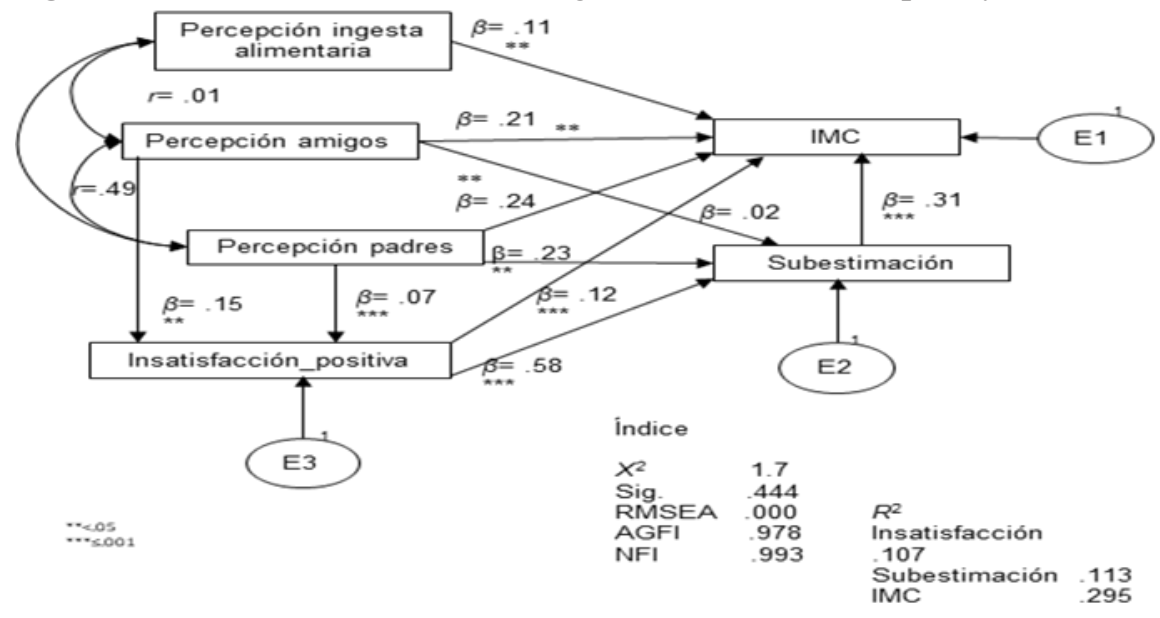

En la misma figura se aprecia que todas las variables, con excepción de "percepción de ingesta alimentaria", que aparece en el lado izquierdo del modelo, mostraron un mismo patrón de respuesta, doble relación directa con IMC y subestimación. Las cargas de las variables "percepción de amigos" y "percepción de padres" son similares ( $\beta$ de
.21 a .24), mientras que la de "insatisfacción positiva" con IMC es baja ( $\beta=.12)$; en cambio, la que se establece con el factor de riesgo "subestimación" es la más alta $(\beta=.58)$.

Debe subrayarse el papel mediador de dos variables: 1) "Insatisfacción positiva", a la que llega la influencia directa de "percepción de amigos" 
y "percepción de padres" para, a partir de allí, influir indirectamente en el IMC y la "subestimación" (percepción de los padres $\rightarrow$ insatisfacción $\rightarrow$ subestimación, y percepción de los padres $\rightarrow$ insatisfacción $\rightarrow$ IMC). Las mismas relaciones se establecen con "percepción de amigos"); 2) "Subestimación", en la que prácticamente confluyen todas las variables del modelo relacionándose de manera directa con el IMC $(\beta=.35)$, que, como se aprecia, es la segunda carga más alta ("percepción de los padres $\rightarrow$ insatisfacción $\rightarrow$ subestimación $\rightarrow$ IMC). Lo mismo ocurre con "percepción de amigos".

La variable "percepción de la ingesta alimentaria" es la única del modelo que solamente tiene una influencia directa con el IMC, aunque con una carga baja $(\beta=.11)$. En la citada figura puede verse que el IMC es la variable que explica el porcentaje más alto de la varianza total (.29\%), además de que $R^{2}=.29$ también indica, de acuerdo con Cohen (1992), el tamaño del efecto, que en este caso es bajo.

El modelo de riesgo para niños identifica seis variables asociadas con el IMC elevado: dieta restringida, deseo de ser más delgado, autopercepción de los padres y de los amigos, inactividad física y subestimación, lo que explica $28 \%$ de la varianza total e indica también un tamaño bajo del efecto. En la Figura 4 se muestra que las variables que influyen más en el IMC de los niños son "subestimación" $(\beta=.43)$ y "percepción de los amigos" $(\beta=.35)$.

Figura 4. Modelo de factores de riesgo en niños con sobrepeso y obesidad.

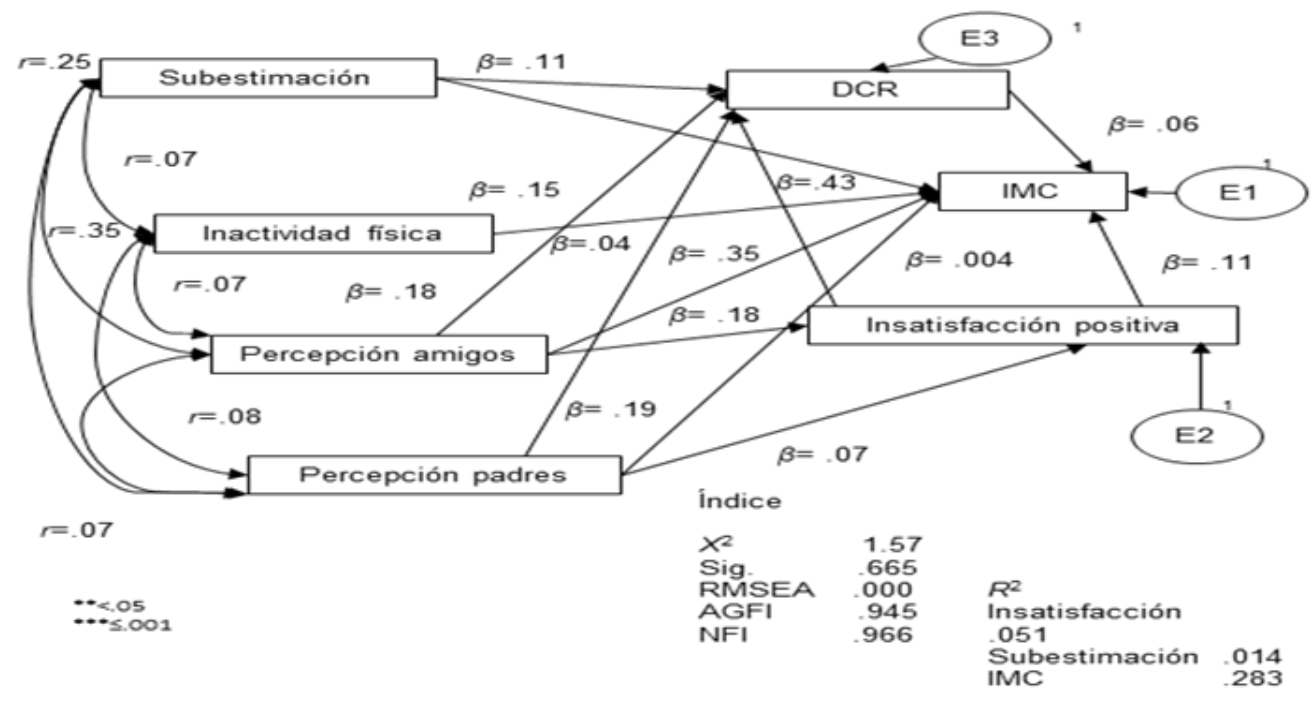

El factor de riesgo "insatisfacción positiva" influye directamente en IMC $(\beta=.11)$, y al mismo tiempo sirve de mediador de las variables "percepción de los amigos" $(\beta=.18)$ y "percepción de los padres" ( $\beta=.07)$ al hacer que éstas influyan indirectamente en el IMC.

La correlación (ligas con doble flecha) entre la percepción de los amigos y la de los padres es la más alta $(\mathrm{r}=.45)$, encontrándose lo mismo en el modelo de las niñas, y prácticamente con igual magnitud (.45 niños vs .49 niñas). Los valores de bondad de ajuste resultaron adecuados, con $X^{2}$ no significativa, RMSEA con dos décimas por arriba de lo esperado, AGFI con una décima por debajo de lo esperado y NFI adecuado (cf. Ruiz, Pardo y San Martín, 2010).

\section{DISCUSIÓN}

El propósito principal del presente estudio fue detectar en una muestra de niños con sobrepeso y obesidad el grado de discordancia o concordancia entre el IMC y la propia percepción del peso corporal. El estudio mostró que tanto los niños como las niñas con exceso de peso corporal se encontraban insatisfechos y con alteración de la imagen corporal; específicamente, subestimaban su peso corporal (tamaño y forma).

El culto al modelo de delgadez, que ha predominado a lo largo de varias décadas y que se ha caracterizado por estigmatizar la obesidad y favorecer un modelo ideal delgado, ha sido internalizado desde edades muy tempranas; los niños 
y prepúberes manifiestan rechazo a las personas obesas. Por ejemplo, en un estudio de Gómez-Peresmitré, Alvarado, Moreno, Saloma y Pineda (2001), niños con un promedio de edad de siete años respondieron a un cuestionario de frases incompletas. En el ítem "Una niña gorda es... ", anotaron términos tales como "fea", "tonta", "prieta" y "asquerosa". En el presente estudio, más de 80\% de los niños y niñas con sobrepeso y obesidad se percibían más delgados, esto es, subestimaban su peso corporal (forma y tamaño). Se encontró que a más peso, más subestimación, resultando alarmante que casi la totalidad de los niños obesos la hicieran. Como era de esperarse, los resultados expuestos muestran que la mayoría de los participantes (más niñas que niños) con exceso de peso corporal se encontraban insatisfechos con su imagen; consecuentemente, los porcentajes de satisfacción fueron bajos y semejantes (20 y 28\%), independientemente del sexo. Resulta interesante que $4 \%$ de los niños con sobrepeso manifestara querer ser más grueso o robusto. Una posible explicación que estudiosos de este tópico han sugerido (Camacho, Escoto, Cedillo y Díaz, 2010; Gómez-Peresmitré et al., 2001, entre otros) es que estos niños podrían querer tener más musculatura y no precisamente estar gordos, por lo que en el futuro se propone llevar a cabo más estudios orientados a resolver esta duda.

Con relación a los modelos estructurales, resulta de gran interés determinar, en el caso del modelo de las niñas con IMC-P85-P95 y $p>95$, la importancia que tienen los factores de riesgo de insatisfacción corporal (deseo de ser más delgadas) y subestimación del peso corporal en relación con el IMC, y confirmar así resultados previamente reportados en algunas investigaciones (GómezPeresmitré et al., 2001; Gómez-Peresmitré et al., 2012). Se ha encontrado también este efecto de subestimación en las madres de los niños obesos, además de que muy frecuentemente desconocen el peso corporal de sus hijos (Guzmán, 2012; Madrigal-Fritsch et al., 1999; Platas, 2014; Vuorela et al., 2010). La importancia de este hallazgo (subestimación del peso corporal) aumenta si se toman en cuenta las implicaciones que puede tener para la gestación y el mantenimiento del sobrepeso y la obesidad durante la pubertad, una etapa de la vida particularmente vulnerable.
Como ya se ha señalado, el efecto de subestimación es definitivamente un factor de riesgo a tenerse en cuenta en los programas de prevención. No debe perderse de vista, además, la influencia que ejercen otras variables en el IMC (ver los modelos de niños y niñas), como la percepción de los amigos y de los padres en la variable de insatisfacción (en el caso del modelo de los niños); debe considerarse que, de acuerdo a Patton, Johnson-Sabine, Wood, Mann y Wakeling (1990) y Gómez-Peresmitré et al. (2001), los efectos de los factores de riesgo se suman.

Entre los niños varones con sobrepeso y obesidad el factor de riesgo más importante fue el de subestimación, aunque también resultó así para las niñas; la diferencia entre unos y otras fue que, en las niñas, la autopercepción de un peso corporal menor del que se tiene sirve de mediadora de la relación con otras variables (percepción de los amigos y de los padres) para influir indirectamente en el IMC. En ambos casos, la influencia de la subestimación del peso corporal con el IMC es directa: a mayor IMC, más delgado se autopercibe el menor.

El papel que desempeña la familia en la aparición de la obesidad resulta controvertido pero trascendental en el desarrollo del niño. No existe una influencia mayor sobre los hábitos alimentarios, actitudes y conductas en torno a los alimentos que la que proviene del ambiente familiar por ser la primera (impronta) y porque es ubicua (presencia latente). No debe perderse de vista que son los padres los modelos o patrones fundamentales de los hijos, por lo que son la base para la formación y modificación de sus conductas. La percepción del peso corporal más próximo a la realidad puede verse influida por las creencias y prácticas culturales, que varían de acuerdo con el nivel socioeconómico y los hábitos alimentarios, tal como señalan Bruss, Morris y Dannison (2003) y González (2009). El hecho de que para los padres de familia el peso no represente un problema sino hasta que se producen alteraciones médicas de gravedad, hace de la obesidad un grave problema de salud pública. Es fundamental trabajar más acerca del significado de los alimentos, que en la mayoría de los casos se utilizan para expresar afecto y cariño, pero también se emplean como un castigo (Orrel-Valente et al., 2007). 
En conclusión, las relaciones más importantes en el modelo de factores de riesgo de sobrepeso y obesidad en las niñas fueron las halladas entre insatisfacción e IMC, insatisfacción y subestimación, y subestimación e IMC. En los niños esas relaciones fueron entre subestimación e IMC, percepción de amigos e IMC, y percepción de amigos e insatisfacción. Valores extremos del IMC (obesidad) relacionados con insatisfacción corporal (deseo de ser más delgado) conducen a una alteración del peso corporal (subestimación).

En cuanto al objeto de interés del presente estudio, se puede señalar que la autopercepción sub- jetiva que conlleva la subestimación del peso contribuye al mantenimiento del exceso de peso corporal en la pubertad. Para la niñez en general, y en especial para las niñas, la subestimación del peso es definitivamente un factor de riesgo que debe tenerse en cuenta en los programas de prevención a desarrollarse en el país. Otro aspecto que debe considerarse en futuros programas de prevención es que no solo debe incluirse en ellos el manejo de hábitos de alimentación saludable y la realización de actividad física en los niños, sino trabajar con los padres para hacerlos conscientes de que la obesidad es una enfermedad.

\section{REFERENCIAS}

Allen, J. y Prkachin, C. G. (2013). Parental awareness and perception of their children's body size. Open Journal of Medical Psychology, 2, 77-80.

Angarita, A., Martínez, A., Rangel, C., Betancourt, M.V. y Prada, G. (2012). Determinantes de la subestimación de la percepción de los padres sobre el peso de sus hijos en hogares infantiles del ICBF de Floridablanca, Colombia. Revista Chilena de Nutriología, 41(4), 372-382.

Arbuckle, J. y Wothke, W. (1999). AMOS users guide version 4.0. Chicago, IL: Small/Waters.

Atalah, E., Urteaga, C. y Rebolledo, A. (2004). Self-perception of nutritional status among adults in Santiago. Revista Médica de Chile, 132(11), 1383-1388.

Barquera, S., Rivera, J., Campos N., I., Hernández B., L., Santos B., C., Durán, E. y Hernández, A.M. (2010). Bases técnicas del acuerdo nacional para la salud alimentaria. Estrategia contra el sobrepeso y la obesidad. México: Secretaria de Salud.

Bruss, M.B., Morris, J. y Dannison, L. (2003). Prevention of childhood obesity: Sociocultural and family factors. Journal of the American Dietetic Association, 103(8), 1042-1045.

Bryteck-Matera, A. (2011). Body attitudes and self-representation in women with eating disorders. Research based on Edward Tory Higgin's self-discrepancy theory. Psychiatria Polska, 45(5), 671-682.

Camacho R., E., Escoto P., C., Cedillo G., C.M. y Díaz C., R. (2010). Correlatos antropométricos de la obsesión por la musculatura. Revista Mexicana de Trastornos Alimentarios, 1(2), 119-124.

Chávez C., K.L., Rodríguez de I., J., Santos G., J., Segovia A., J.G., Altamirano M., D.C. y Matías B., V.M. (2016). Alteración de la percepción del estado nutricional por parte de padres de preescolares: un factor para obesidad y sobrepeso. Archivos Argentinos de Pediatría, 114(3), 237-242.

Chen, S., Binns, C.W., Zhao, Y. y Liu, Y. (2014). Chinese mothers' perceptions of their child's weight and obesity status. Asia Pacific Journal of Clinical Nutrition, 23(3), 452-458.

Cohen, J. (1992). A power primer. Psychological Bulletin, 112(1), 155-159.

Córdoba V., J.Á. (2016). La obesidad: la verdadera pandemia del siglo XXI. Cirugía y Cirujanos, 84(5), 351-355.

Gómez-Peresmitré, G. (1997). Alteraciones de la imagen corporal en una muestra de escolares mexicanos preadolescentes. Revista Mexicana de Psicología, 14, 31-40.

Gómez-Peresmitré, G. (2001). Factores de riesgo en trastornos de la conducta alimentaria. Teoría práctica y prevalencia en muestras mexicanas. Memorias de la Presentación de los 450 años de la Universidad. México: UNAM.

Gómez-Peresmitré, G., Alvarado, G., Moreno, L., Saloma, S. y Pineda, G. (2001). Trastornos de la alimentación. Factores de riesgo en tres diferentes grupos de edad: prepúberes, púberes y adolescentes. Revista Mexicana de Psicología, 18, $313-324$.

Gómez-Peresmitré, G., Jaëger, B., Pineda, G. y Platas A., S. (2012). Comparing body image and risk eating behavior between Mexican and German women. Revista Mexicana de Trastornos Alimentarios, 3(1), 19-28.

Gómez-Peresmitré, G., Platas A., S. y Pineda G., G. (2015). A preliminary study of an online self-detection test. Risk in eating disorders. Revista Mexicana de Trastornos Alimentarios, 6(1), 22-29.

Gómez-Peresmitré, G., Platas A., S., Pineda G., G. y Guzmán S., R. (en revisión). Programa de prevención para la obesidad en escolares. Revista de Psicología Clínica en Niños y Adolescentes. 
González W., J. (2009). Características socioeconómicas, familiares y ambientales en niños obesos de la parroquia Antimano, Caracas. Revista de Ciencias Sociales, 15(2), 235-244.

Guzmán S., R.M.E. (2012). Factores psicosociales asociados con conductas alimentarias de riesgo en adolescentes con obesidad. Tesis inédita de Doctorado en Psicología. México: UNAM.

Hernández, B., Gortmaker, S., Liard, N., Colditz, G., Parra C., S. y Peterson, K. (2000). Validez y reproductibilidad de un cuestionario de actividad e inactividad física para escolares de la Ciudad de México. Salud Pública de México, 42(4), $315-323$.

Jáuregui L., I., Bolaños R., P., Santiago F., M.J. y Garrido C., O. (2011). Perception of weight and phychological variables in a simple of Spanish adolescents. Diabetes, Metabolic Syndrome and Obesity: Targets and Therapy, 4, $245-251$.

Lundahl, A., Kidwell, K.M. y Nelson, T.D. (2014). Parental underestimates of child weight: A meta-analysis. Pediatrics, 133(3), $1-15$.

Madrigal F., H., De Irala E., J., Martínez G., M.A., Kearney, J., Gibney, M. y Martínez H., J.A. (1999). The perception of body image as a qualitative approach to nutritional status. Salud Pública de México, 41(6), 479-486.

Madrigal F., H., Sánchez V., A., Martínez G., M.A., Kearney, J., Gibney, M.J., De Irala, J. y Martínez, J.A. (2000). Underestimation of body mass index through perceived body image as compared to self-reported body mass index in the European Union. Public Health, 114(6), 468-473.

Matus L., N.P., Álvarez G., G.C., Nazar B., D.A. y Mondragón R., R. (2016). Percepciones de adultos con sobrepeso y obesidad y su influencia en el control de peso en San Cristóbal de Las Casas, Chiapas. Estudios Sociales, 24(47), 380-409.

Míguez B., M., De la Montaña M., J., González C., J. y González R., M. (2011). Agreement between the self-perception on the body image and the nutritional status in college students from Orense. Nutrición Hospitalaria, 26(3), $472-479$.

Mújica E., V., Leiva M., E., Rojas M., E., Díaz, N., Icaza, G. y Palomo G., I. (2009). Discordancia en autopercepción de peso en población adulta de Talca. Revista Médica de Chile, 137(1), 76-82.

National Center for Health Statistics (2003). Overweight and obesity statistics. Hyatsville, MD: NCHS. Recuperado de https:// www.cdc.gov/nchs/pressroom/06facts/obesity03_04.htm.

Organización Mundial de la Salud (2016). Obesidad y sobrepeso. Ginebra: OMS. Recuperado de http://www.who.int/mediacentre/ factsheets/fs311/es/.

Orrell-Valente, J.K., Hill, L.G., Brechwald, W.A., Dodge, K.A., Pettit, G.S. y Bates, J.E. (2007). Just three more bites: An observational analysis of parent's socialization of children's eating at mealtime. Appetite, 48(1), 37-45.

Park, M. (2014). Half of parents underestimate the weight of their overweight or obese children. Evidence Based Nursing, 18, 2, 47. doi: 10.1136/eb-2014-101876.

Pasch, L.A., Penilla, C., Tschann, J.M., Martínez, S.M., Deardorff, J., De Groat, C.L. y Greenspan, L.C. (2016). Preferred child body size and parental underestimation of child weight in Mexican-American families. Maternal and Child Health Journal, 20(9), 1842-1848.

Patton, G.C., Johnson-Sabine, E., Wood, K., Mann, A.H. y Wakeling, A. (1990). Abnormal eating attitudes in London schoolgirls a prospective epidemiological study: Outcome at twelve-month follow-up. Psychological Medicine, 20(2), $383-394$.

Platas A., R.S. (2014). Modelo de factores protectores y de riesgo para los problemas de peso corporal en escolares de primaria. Tesis inédita de Doctorado en Psicología. México: UNAM.

Platas A., R.S. y Gómez-Peresmitré, G. (2013). Propiedades psicométricas de la Escala de Factores de Riesgo Asociados con Trastornos de la Alimentación (EFRATA-II) en preadolescentes mexicanos. Psicología y Salud, 23(2), $251-259$.

Ruiz, M.A., Pardo, A. y San Martin, R. (2010). Modelos de ecuaciones estructurales. Papeles del Psicólogo, 31(1), 34-45.

Sociedad Mexicana de Psicología (2010). Código ético del psicólogo. México: Trillas.

Vuorela, N., Saha, M.T. y Salo, M.K. (2010). Parent underestimates their child's overweight. Acta Pediátrica, 99(9), 1374-1379.

West, D., Raczynski, J., Phillips, M., Bursac, Z., Gauss. H. y Montgomery, B. (2008). Parental recognition of overweight in school-age children, Obesity, 16(3), 630-636.

Zamudio S., O. y Muñoz A., R.M. (2016). Percepción de los padres respecto al sobrepeso y obesidad de sus hijos. Revista de Enfermería del Instituto Mexicano del Seguro Social, 24(1), 61-64.

Zonana N., A. y Conde G., M.E. (2010). Percepción de las madres sobre la obesidad de sus hijos. Gaceta Médica de México, $146(3), 165-168$. 